\title{
Smooth Fano Polytopes Whose Ehrhart Polynomial Has a Root with Large Real Part
}

\author{
Hidefumi Ohsugi · Kazuki Shibata
}

Received: 5 September 2011 / Revised: 2 November 2011 / Accepted: 13 January 2012 /

Published online: 3 February 2012

(C) Springer Science+Business Media, LLC 2012

\begin{abstract}
The symmetric edge polytopes of odd cycles (del Pezzo polytopes) are known as smooth Fano polytopes. In this paper, we show that if the length of the cycle is 127 , then the Ehrhart polynomial has a root whose real part is greater than the dimension. As a result, we have a smooth Fano polytope that is a counterexample to the two conjectures on the roots of Ehrhart polynomials.
\end{abstract}

Keywords Ehrhart polynomials · Gröbner bases · Gorenstein Fano polytopes

\section{Introduction}

Let $d \geq 3$ be an integer, and $A_{d}$ the $(d+1) \times(2 d+1)$ matrix

$$
A_{d}=\left(\begin{array}{c|cccc|cccc}
0 & 1 & & & -1 & -1 & & & 1 \\
0 & -1 & \ddots & & & 1 & \ddots & & \\
\vdots & & \ddots & 1 & & & \ddots & -1 & \\
0 & & & -1 & 1 & & & 1 & -1 \\
\hline 1 & 1 & \cdots & 1 & 1 & 1 & \cdots & 1 & 1
\end{array}\right) .
$$

In this paper, we study the convex hull $\operatorname{Conv}\left(A_{d}\right)$ of $A_{d}$. The matrix $A_{d}$ is the centrally symmetric configuration [6], and $\operatorname{Conv}\left(A_{d}\right)$ is called the symmetric edge polytope of the cycle of length $d$. From the results in $[3,5]$ we have the following:

H. Ohsugi $(\bowtie) \cdot$ K. Shibata

Department of Mathematics, College of Science, Rikkyo University, Toshima-ku, Tokyo 171-8501, Japan

e-mail: ohsugi@rikkyo.ac.jp

K. Shibata

e-mail: 101c002r@rikkyo.ac.jp 
Proposition The polytope $\operatorname{Conv}\left(A_{d}\right)$ is a Gorenstein Fano polytope (reflexive polytopes) of dimension $d-1$. In addition, $\operatorname{Conv}\left(A_{d}\right)$ is a smooth Fano polytope if and only if $d$ is odd.

Here, we first construct the reduced Gröbner basis $\mathcal{G}$ of $I_{A_{d}}$. Next, using $\mathcal{G}$, we compute the Ehrhart polynomial and the $h$-vector of $\operatorname{Conv}\left(A_{d}\right)$. Finally, we study the roots of the Ehrhart polynomial when $d$ is odd. We show that the Ehrhart polynomial of $\operatorname{Conv}\left(A_{127}\right)$ has a root whose real part is greater than $\operatorname{dim}\left(\operatorname{Conv}\left(A_{127}\right)\right)$. This is a counterexample to the conjectures given in $[1,5]$.

\section{Gröbner Bases of Toric Ideals}

Let $\mathcal{R}_{d}=K\left[t_{1}, t_{1}^{-1}, \ldots, t_{d}, t_{d}^{-1}, s\right]$ be the Laurent polynomial ring over a field $K$, and let $K[z, X, Y]=K\left[z, x_{1}, \ldots, x_{d}, y_{1}, \ldots, y_{d}\right]$ be the polynomial ring over $K$. We define the ring homomorphism $\pi: K[z, X, Y] \rightarrow \mathcal{R}_{d}$ by setting $\pi\left(x_{i}\right)=t_{i} t_{i+1}^{-1} s$, $\pi\left(y_{i}\right)=t_{i}^{-1} t_{i+1} s$ for $1 \leq i \leq d$ (here we set $\left.t_{d+1}=t_{1}\right)$, and $\pi(z)=s$. The toric ideal $I_{A_{d}}$ is $\operatorname{ker}(\pi)$. Let $<$ be the reverse lexicographic order on $K[z, X, Y]$ with ordering $z<y_{d}<x_{d}<\cdots<y_{1}<x_{1}$. For $d \geq 3$, let $[d]=\{1, \ldots, d\}$ and $k=\left\lceil\frac{d}{2}\right\rceil$.

Theorem 2.1 The reduced Gröbner basis of $I_{A_{d}}$ with respect to $<$ consists of

$$
\begin{aligned}
& x_{i} y_{i}-z^{2} \quad(1 \leq i \leq d), \\
& \prod_{l=1}^{k} x_{i_{l}}-z \prod_{l=1}^{k-1} y_{j_{l}} \quad\left([d]=\left\{i_{1}, \ldots, i_{k}\right\} \cup\left\{j_{1}, \ldots, j_{k-1}\right\}\right), \\
& \prod_{l=1}^{k} y_{j_{l}}-z \prod_{l=1}^{k-1} x_{i_{l}} \quad\left([d]=\left\{i_{1}, \ldots, i_{k-1}\right\} \cup\left\{j_{1}, \ldots, j_{k}\right\}\right)
\end{aligned}
$$

if $d$ is odd and

$$
\begin{aligned}
& x_{i} y_{i}-z^{2} \quad(1 \leq i \leq d), \\
& \prod_{l=1}^{k} x_{i_{l}}-y_{d} \prod_{l=1}^{k-1} y_{j_{l}} \quad\left([d-1]=\left\{i_{1}, \ldots, i_{k}\right\} \cup\left\{j_{1}, \ldots, j_{k-1}\right\}\right), \\
& \prod_{l=1}^{k} y_{j_{l}}-x_{d} \prod_{l=1}^{k-1} x_{i_{l}} \quad\left([d-1]=\left\{i_{1}, \ldots, i_{k-1}\right\} \cup\left\{j_{1}, \ldots, j_{k}\right\}\right)
\end{aligned}
$$

if $d$ is even. The initial monomial of each binomial is the first monomial.

Proof Let $\mathcal{G}$ be the set of all binomials above. It is easy to see that $\mathcal{G} \subset I_{A_{d}}$ and that the initial monomial of each binomial in $\mathcal{G}$ is the first monomial. Let in $(\mathcal{G})=$ $\left\langle\right.$ in $\left._{<}(g) \mid g \in \mathcal{G}\right\rangle$. Suppose that $d$ is odd and that $\mathcal{G}$ is not a Gröbner basis of $I_{A_{d}}$. Then, there exists an irreducible binomial $(0 \neq) f=u-v \in I_{A_{d}}$ such that neither $u$ nor $v$ belongs to in $(\mathcal{G})$. Let $u=z^{\alpha} \prod_{l=1}^{m} x_{i_{l}}^{p_{l}} \prod_{l=1}^{n} y_{j_{l}}^{q_{l}}$ and $v=z^{\alpha^{\prime}} \prod_{l=1}^{m^{\prime}} x_{i_{l}^{\prime}}^{p_{l}^{\prime}} \prod_{l=1}^{n^{\prime}} y_{j_{l}^{\prime}}^{q_{l}^{\prime}}$, 
where $0<p_{l}, q_{l}, p_{l}^{\prime}, q_{l}^{\prime} \in \mathbb{Z}$ for all $l$, and $\mathcal{I}=\left\{i_{1}, \ldots, i_{m}\right\}, \mathcal{J}=\left\{j_{1}, \ldots, j_{n}\right\}, \mathcal{I}^{\prime}=$ $\left\{i_{1}^{\prime}, \ldots, i_{m^{\prime}}^{\prime}\right\}$, and $\mathcal{J}^{\prime}=\left\{j_{1}^{\prime}, \ldots, j_{n^{\prime}}^{\prime}\right\}$ are subsets of $[d]$ with cardinalities $m, n, m^{\prime}$, and $n$, respectively. Since neither $u$ nor $v$ is divided by $x_{i} y_{i}$, we have $\mathcal{I} \cap \mathcal{J}=\mathcal{I}^{\prime} \cap \mathcal{J}^{\prime}=\emptyset$. In addition, since neither $u$ nor $v$ is divided by the initial monomials of binomials (2) and (3), it follows that $m, n, m^{\prime}, n^{\prime} \leq k-1$. Moreover, since $f$ is irreducible, we have $\mathcal{I} \cap \mathcal{I}^{\prime}=\mathcal{J} \cap \mathcal{J}^{\prime}=\emptyset$. Let $p=\sum_{l=1}^{m} p_{l}, q=\sum_{l=1}^{n} q_{l}, p^{\prime}=\sum_{l=1}^{m^{\prime}} p_{l}^{\prime}$, and $q^{\prime}=\sum_{l=1}^{n^{\prime}} q_{l}^{\prime}$. Then, $\pi(u)=s^{\alpha+p+q} \prod_{l=1}^{m}\left(t_{i_{l}} t_{i_{l}+1}^{-1}\right)^{p_{l}} \prod_{l=1}^{n}\left(t_{j_{l}}^{-1} t_{j_{l}+1}\right)^{q_{l}}$ and $\pi(v)=$ $s^{\alpha^{\prime}+p^{\prime}+q^{\prime}} \prod_{l=1}^{m^{\prime}}\left(t_{i_{l}^{\prime}} t_{i_{l}^{\prime}+1}^{-1}\right)^{p_{l}^{\prime}} \prod_{l=1}^{n^{\prime}}\left(t_{j_{l}^{\prime}}^{-1} t_{j_{l}^{\prime}+1}\right)^{q_{l}^{\prime}}$, where we set $t_{d+1}=t_{1}$. Since $\pi(u)=$ $\pi(v)$, it follows that $\pi\left(u^{\prime}\right)=\pi\left(v^{\prime}\right)$, where $u^{\prime}=z^{\alpha+2 q} \prod_{l=1}^{m} x_{i_{l}}^{p_{l}} \prod_{l=1}^{n^{\prime}} x_{j_{l}^{\prime}}^{q_{l}^{\prime}}$ and $v^{\prime}=$ $z^{\alpha^{\prime}+2 q^{\prime}} \prod_{l=1}^{m^{\prime}} x_{i_{l}^{\prime}}^{p_{l}^{\prime}} \prod_{l=1}^{n} x_{j_{l}}^{q_{l}}$. Thus, $g=u^{\prime}-v^{\prime}$ belongs to $I_{A_{d}}$. Since $g$ belongs to $K[z, X], g$ belongs to the toric ideal $I_{B}$, where $B$ is the matrix consisting of the first $d+1$ columns of $A_{d}$. In addition, by virtue of $m, n, m^{\prime}, n^{\prime} \leq k-1$, we have $\left|\mathcal{I} \cup \mathcal{J}^{\prime}\right| \leq 2(k-1)<d$ and $\left|\mathcal{I}^{\prime} \cup \mathcal{J}\right| \leq 2(k-1)<d$. Thus, neither $u^{\prime}$ nor $v^{\prime}$ is divided by $x_{1} \cdots x_{d}$. Since $g \in I_{B}=\left\langle x_{1} \cdots x_{d}-z^{d}\right\rangle$, we have $g=0$, that is, $u^{\prime}=v^{\prime}$. Then, from $\mathcal{I} \cap \mathcal{J}=\mathcal{I}^{\prime} \cap \mathcal{J}^{\prime}=\mathcal{I} \cap \mathcal{I}^{\prime}=\mathcal{J} \cap \mathcal{J}^{\prime}=\emptyset$ we have $\left(\mathcal{I} \cup \mathcal{J}^{\prime}\right) \cap\left(\mathcal{I}^{\prime} \cup \mathcal{J}\right)=\emptyset$. Hence, $\prod_{l=1}^{m} x_{i_{l}}^{p_{l}} \prod_{l=1}^{n^{\prime}} x_{j_{l}^{\prime}}^{q_{l}^{\prime}}$ and $\prod_{l=1}^{m^{\prime}} x_{i_{l}^{\prime}}^{p_{l}^{\prime}} \prod_{l=1}^{n} x_{j_{l}}^{q_{l}}$ have no common variables. Since $u^{\prime}=v^{\prime}$, we have $m=n=m^{\prime}=n^{\prime}=0$. Hence, $u=z^{\alpha}$ and $v=z^{\alpha^{\prime}}$. Since $f$ is a homogeneous binomial, this is a contradiction. Thus, $\mathcal{G}$ is a Gröbner basis of $I_{A_{d}}$. It is trivial that $\mathcal{G}$ is reduced. The case where $d$ is even is analyzed by a similar argument.

\section{Ehrhart Polynomials and Roots}

For $0 \leq i \leq d$, let $r_{d}(i)$ denote the number of square-free monomials in $K[X, Y]$ of degree $i$ that do not belong to the initial ideal in $\operatorname{in}_{<}\left(I_{A_{d}}\right)$, and let $s_{d}(i+1)$ denote the number of square-free monomials in $K[z, X, Y]$ of degree $(i+1)$ that are divided by $z$ and do not belong to $\operatorname{in}_{<}\left(I_{A_{d}}\right)$. For example, $r_{d}(0)=s_{d}(1)=1$ and $r_{d}(d)=0$.

Lemma 3.1 For $0 \leq i \leq d-1$, we have $r_{d}(i)=\left(\begin{array}{c}d \\ i\end{array}\right) \sum_{\ell=1}^{d-i}\left(\begin{array}{c}i \\ k-\ell\end{array}\right)$ and $s_{d}(i+1)=$ $r_{d}(i)$. In particular, $r_{d}(i)=\left(\begin{array}{c}d \\ i\end{array}\right) 2^{i}$ for $0 \leq i \leq k-1$.

Proof Since the variable $z$ does not appear in the initial monomials of the binomials in Theorem 2.1, $u \notin \operatorname{in}_{<}\left(I_{A_{d}}\right)$ if and only if $z u \notin$ in $_{<}\left(I_{A_{d}}\right)$ for any square-free monomial $u \in K[X, Y]$. Thus, $s_{d}(i+1)=r_{d}(i)$ for $0 \leq i \leq d-1$. Suppose that $d$ is odd. Then, by Theorem 2.1, $r_{d}(i)$ is the number of monomials $\prod_{i \in \mathcal{I}} x_{i} \prod_{j \in \mathcal{J}} y_{j}$ where $\mathcal{I}, \mathcal{J} \subset[d], \mathcal{I} \cap \mathcal{J}=\emptyset,|\mathcal{I} \cup \mathcal{J}|=i$, and $|\mathcal{I}|,|\mathcal{J}| \leq k-1$. Since the number of subsets $\mathcal{I}, \mathcal{J} \subset[d]$ such that $\mathcal{I} \cap \mathcal{J}=\emptyset,|\mathcal{I} \cup \mathcal{J}|=i$, and $|\mathcal{I}|=\lambda$ is $\left(\begin{array}{c}d \\ \lambda, i-\lambda, d-i\end{array}\right)=\left(\begin{array}{c}d \\ d-i\end{array}\right)\left(\begin{array}{l}i \\ \lambda\end{array}\right)=$ $\left(\begin{array}{c}d \\ i\end{array}\right)\left(\begin{array}{c}i \\ \lambda\end{array}\right)$, it follows that $r_{d}(i)=\sum_{\ell=1}^{d-i}\left(\begin{array}{c}d \\ i\end{array}\right)\left(\begin{array}{c}i \\ k-\ell\end{array}\right)=\left(\begin{array}{c}d \\ i\end{array}\right) \sum_{\ell=1}^{d-i}\left(\begin{array}{c}i \\ k-\ell\end{array}\right)$ for $0 \leq i \leq d-1$. If $d$ is even, then the proof is similar. 
It is known [9, Chap. 8] that $\operatorname{in}_{<}\left(I_{A_{d}}\right)=\sqrt{\operatorname{in}_{<}\left(I_{A_{d}}\right)}$ is the Stanley-Reisner ideal of a regular unimodular triangulation $\Delta$ of $\operatorname{Conv}\left(A_{d}\right)$. Thus, $r_{d}(i)+r_{d}(i+1)$ is the number of $i$-dimensional faces of $\Delta$. From Lemma 3.1 and [7, Theorem 1.4], the Hilbert polynomial of $K[z, X, Y] / I_{A_{d}}$ can be computed as follows:

Theorem 3.2 The Ehrhart polynomial of $\operatorname{Conv}\left(A_{d}\right)$ is $\sum_{i=0}^{d-1} r_{d}(i)\left(\begin{array}{c}m \\ i\end{array}\right)$. Moreover, the normalized volume of $\operatorname{Conv}\left(A_{d}\right)$ equals $k\left(\begin{array}{l}d \\ k\end{array}\right)$.

Let $\left(h_{0}^{(d)}, h_{1}^{(d)}, \ldots, h_{d-1}^{(d)}\right)$ be the $h$-vector of $\operatorname{Conv}\left(A_{d}\right)$. Note that $h_{0}^{(d)}=1$. Since $\operatorname{Conv}\left(A_{d}\right)$ is Gorenstein, we have $h_{j}^{(d)}=h_{d-1-j}^{(d)}$ for each $0 \leq j \leq d-1$. Thus, it is enough to study $h_{j}^{(d)}$ for $1 \leq j \leq k-1$.

Theorem 3.3 For $1 \leq j \leq k-1$, we have

$$
\begin{aligned}
h_{j}^{(d)} & =(-1)^{j} \sum_{i=0}^{j}(-2)^{i}\left(\begin{array}{l}
d \\
i
\end{array}\right)\left(\begin{array}{c}
d-i-1 \\
j-i
\end{array}\right) \\
& = \begin{cases}2^{d-1} & j=k-1 \text { and } d \text { is odd }, \\
h_{j}^{(d-1)}+h_{j-1}^{(d-1)} & \text { otherwise. }\end{cases}
\end{aligned}
$$

Proof By Lemma 3.1 and a well-known expression [7, p. 58], one can show the first equality and that $h_{j}^{(d)}$ is the coefficient of $u^{j}$ in the expansion of $2^{d}(u+1)^{d} \times$ $(u+2)^{-d+j}$. The second equality follows from this fact and the identity given in $[8$, p. 148].

Finally, we study the roots of the Ehrhart polynomial when $d$ is odd. In this case, $\operatorname{Conv}\left(A_{d}\right)$ is a smooth Fano polytope of dimension $d-1$. Since $\operatorname{Conv}\left(A_{d}\right)$ is a Gorenstein Fano polytope, the roots of the Ehrhart polynomial are symmetrically distributed in the complex plane with respect to the line $\operatorname{Re}(z)=-1 / 2$. Here, $\operatorname{Re}(z)$ is the real part of $z \in \mathbb{C}$. The following conjectures are given in $[1,5]$.

Conjecture 3.4 [1] All roots $\alpha$ of Ehrhart polynomials of $D$-dimensional lattice polytopes satisfy $-D \leq \operatorname{Re}(\alpha) \leq D-1$.

Conjecture 3.5 [5] All roots $\alpha$ of Ehrhart polynomials of $D$-dimensional Gorenstein Fano polytopes satisfy $-D / 2 \leq \operatorname{Re}(\alpha) \leq D / 2-1$.

Using the software packages Maple, Mathematica, and Maxima, we computed the largest real part of roots of the Ehrhart polynomial of $\operatorname{Conv}\left(A_{d}\right)$ (see Table 1).

Remark 3.6 It was shown [2] that Conjecture 3.4 is true for $D \leq 5$. Recently, a simplex (not a Fano polytope) that does not satisfy the condition " $\operatorname{Re}(\alpha) \leq D-1$ " in Conjecture 3.4 was presented in [4]. Our polytope $\operatorname{Conv}\left(A_{125}\right)$ is the first example satisfying neither " $-D \leq \operatorname{Re}(\alpha)$ " nor " $\operatorname{Re}(\alpha) \leq D-1$ " in Conjecture 3.4. 
Table 1 The largest real part of roots of the Ehrhart polynomial

\begin{tabular}{rlll}
\hline$d$ & $\operatorname{dim}\left(\operatorname{Conv}\left(A_{d}\right)\right)$ & The largest real part & \\
\hline 35 & 34 & 16.35734046 & A counterexample to Conjecture 3.5 \\
125 & 124 & 123.5298262 & A counterexample to Conjecture 3.4 \\
127 & 126 & 126.5725840 & Greater than its dimension \\
\hline
\end{tabular}

Acknowledgements The authors are grateful to Takayuki Hibi, Akihiro Higashitani, and Tetsushi Matsui for useful discussions. This research was supported by JST CREST.

\section{References}

1. Beck, M., De Loera, J.A., Develin, M., Pfeifle, J., Stanley, R.P.: Coefficients and roots of Ehrhart polynomials. In: Integer points in polyhedra-geometry, number theory, algebra, optimization. Contemp. Math. 374, 15-36 (2005)

2. Braun, B., Develin, M.: Ehrhart polynomial roots and Stanley's non-negativity theorem. In: Integer points in polyhedra - geometry, number theory, representation theory, algebra, optimization, statistics. Contemp. Math. 452, 67-78 (2008)

3. Higashitani, A.: Smooth Fano polytopes arising from finite directed graphs, preprint. arXiv:1103. 2202v1 [math.CO]

4. Higashitani, A.: Counterexamples of the conjecture on roots of Ehrhart polynomials. Discrete Comput. Geom. doi:10.1007/s00454-011-9390-4

5. Matsui, T., Higashitani, A., Nagazawa, Y., Ohsugi, H., Hibi, T.: Roots of Ehrhart polynomials arising from graphs. J. Algebr. Comb. 34, 721-749 (2011)

6. Ohsugi, H., Hibi, T.: Centrally symmetric configurations of integer matrices, preprint. arXiv: 1105.4322v1 [math.AC]

7. Stanley, R.P.: Combinatorics and Commutative Algebra, 2nd edn. Progress in Mathematics, vol. 41. Birkhäuser Boston, Boston (1996)

8. Stanley, R.P.: Enumerative Combinatorics, vol. 2. Cambridge University Press, Cambridge (1999)

9. Sturmfels, B.: Gröbner Bases and Convex Polytopes. Am. Math. Soc., Providence (1996) 